\title{
Strategy for Processing and Analyzing Social Media Data Streams in Emergencies
}

\author{
Matthias Moi, Therese Friberg and Robin Marterer \\ C.I.K. \\ University of Paderborn \\ Paderborn, Germany \\ \{moi;friberg;marterer\}@cik.uni-paderborn.de
}

\author{
Christian Reuter and Thomas Ludwig \\ Institute for Information Systems \\ University of Siegen \\ Siegen, Germany \\ \{christian.reuter; thomas.ludwig\}@uni-siegen.de
}

\author{
Deborah Markham, Mike Hewlett and Andrew Muddiman \\ Oxford Computer Consultants Ltd \\ Oxford, UK \\ \{deborah.markham; mike.hewlett;andrew.muddiman\}@oxfordcc.co.uk
}

\begin{abstract}
People are using social media to a greater extent, particularly in emergency situations. However, approaches for processing and analyzing the vast quantities of data produced currently lag far behind. In this paper we discuss important steps, and the associated challenges, for processing and analyzing social media in emergencies. In our research project EmerGent, a huge volume of low-quality messages will be continuously gathered from a variety of social media services such as Facebook or Twitter. Our aim is to design a software system that will process and analyze social media data, transforming the high volume of noisy data into a low volume of rich content that is useful to emergency personnel. Therefore, suitable techniques are needed to extract and condense key information from raw social media data, allowing detection of relevant events and generation of alerts pertinent to emergency personnel.
\end{abstract}

Keywords - social media; information gathering; information mining; ontology; emergency services; emergencies; information quality; information visualisation

\section{INTRODUCTION AND PROBLEM DESCRIPTION}

Social media (SM) is a fast growing phenomenon for creating and maintaining social links. The convergence of social networking and mobile technology is shifting the way people communicate, as well as their methods for gaining and sharing information. During disasters throughout the world, such as the 2013 European Floods [1], 2013 Colorado floods [2], or 2012 Hurricane Sandy [3], SM has been widely used. For example, the term "Sandy" generated over 3.4 million mentions across Facebook and Twitter

Information available on SM is rapidly becoming an important source for enhancing situation awareness [4]. An important challenge we face within our work is to transform a high-volume of data, much of which is irrelevant or duplicate, from SM into a low-volume of rich information for recipients. Emergency services, such as the fire or police departments, currently do not have the resources to use potentially emergency-related information from SM, thus risking the loss of essential information in an emergency [5]. The development of adequate methodologies and techniques from many fields, including those of data mining and information quality, are essential for us to resolve this dilemma [6].

This work is part of the research project EmerGent. This project aims to analyse the positive and negative impact of SM in emergencies (by using the developed software system as an instrument), to enhance the objective and perceived safety and security of citizens before, during and after emergencies. Moreover, this project will strengthen the role of European companies dealing with services and products related to the results. In this paper, we discuss the key steps we plan to incorporate into our software system for processing and analysing SM data streams in emergencies. We begin by discussing each of the main stages of our system, in their logical order. Thereafter we discuss some preliminary visualisation techniques for handling the amount of data, before concluding with a broad outlook as well as a discussion of further work.

\section{IMPORTANT STEPS FOR PROCESSING AND ANALYZING SOCIAL MEDIA STREAMS}

According to the crisis communication matrix in [7] we generally distinguish between four main directions of communication: (1) communication between citizens (C2C), (2) between authorities (A2A) and (3) from citizens to authorities $(\mathrm{C} 2 \mathrm{~A})$ and $(4)$ vice versa (A2C). For the discussion in this paper we are focusing on the communication that occurs via SM from citizens to authorities and the communication between citizens $(\mathrm{C} 2 \mathrm{~A}$ and $\mathrm{C} 2 \mathrm{C})$. These communications are most relevant when processing data for emergency services, because it is currently not possible for emergency services to efficiently use this information without some automated processing in place. Furthermore, especially in the communication between citizens, relevant unusual events or warnings may occur before, during and after emergencies, allowing emergency services to track the full lifecycle of an emergency.

A simplified process for obtaining useful information from $\mathrm{SM}$ is shown in Fig. 1. After the selection of different data sources and SM services, data must be gathered and then 
adjusted to obtain homogeneous data sets. In the next step, the slightly pre-processed data is enriched by the application of approaches to extract important meta-data like date/time, author or language from the content, but also further implicit information like the sentiment of social media posts. Based on the enriched data a deeper analysis, such as semantic analysis, can be applied to identify relevant information.

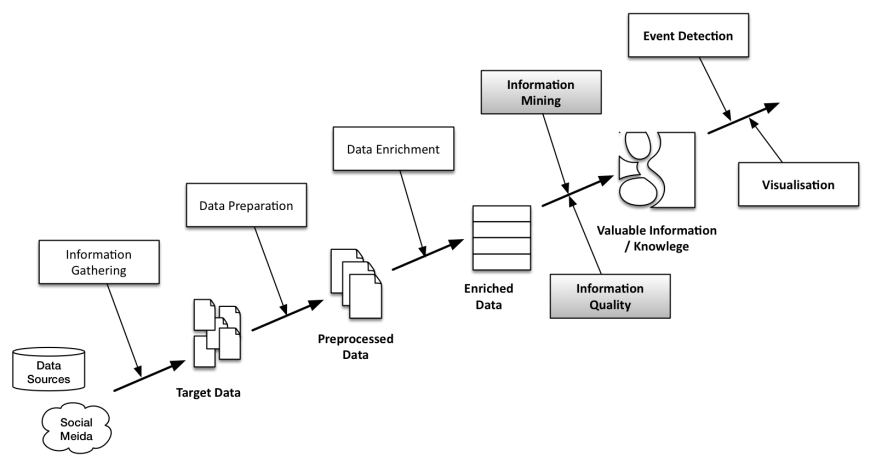

Fig. 1. Simplified process for processing and analyzing SM data

\section{A. Information Gathering and Data Preparation}

Key challenges when gathering and analyzing SM are the diversity of SM services and the presence of different data formats (e.g. a tweet in Twitter or a video in YouTube). Therefore, to allow further processing of the heterogeneous SM information we need to standardize the exchange data format. There are already approaches existing that attempt to harmonize multiple SM services on a data level. Due to their wide usage and deployment the OpenSocial ${ }^{1}$ API formats deserve a closer inspection.

OpenSocial specifies an Activity Streams format whereby an activity is defined as "a semantic description of potential or completed actions", which has at least a verb (the type of activity, e.g. like, post, share), an actor (e.g. the creator) and an object (e.g. an image or message object), but may also contain a target, participants and further attributes. There are already multiple verbs and object types defined within the specification. For instance, a Place object may contain the attributes latitude, longitude and altitude. Although the specification allows modeling the activities of liking, sharing and so on; there are no attributes designated to carry information such as "20 users shared/liked this post", which need to be implemented in the post-processing application. Activity objects must be encapsulated in a Collection object before serializing and returning them as a $\mathrm{JSON}^{2}$ object. The benefit of the exchange format of Activity Streams is that each client, which will be built upon Activity Streams, can automatically process the output. The Activity Streams 2.0 Action Handlers specification provides standard mechanisms allowing client applications to perform actions (e.g. like or share a video) without prior knowledge of the underlying architecture.

Other challenges in gathering social media data are the different technical and business-oriented limitations [8]. For example, many sites have rate limits for freely available data. Of course these restrictions can be lifted for a cost, but this depends on the income restriction of users.

\section{B. Data Enrichment}

Various studies indicate that extraction of relevant information is a major challenge [9], [10]. Different circumstances require different assessment methods [11], and different data or meta-data. We can distinguish between source-based and computation-based enriched data: sourcebased data is either directly given by the raw data, or not provided, and therefore requires no further computation besides extraction. The actual source-based data varies among different social networks such as Facebook, Google+, Instagram, Twitter or YouTube and is restricted by different technical and business-oriented limitations [8]. Provided data includes date, time, sender, title, tags, keywords, comments, replies, answers, number of views, dislikes, retweets, shares, age, gender, location, education, uploads, watches, total posts and real name. In addition to source-based enriched data, we will provide computation-based enriched data, which requires one or multiple steps of algorithmic computation. Whilst some of the computations can be done on the local server, others may require the invocation of remote APIs. Computationally obtained data includes language detection and sentiment analysis [12].

Related work has been carried out in this field: [13] collects data with useful features (like from Facebook and Twitter) and analyzes them to add new features (e.g. sentiment score); [14] presents a graph-based model of contributor relationships and combines it with content and usage based features to exploit community feedback (such as links and explicit quality ratings from members) to automatically identify high quality content in "Yahoo! Answers". Answer length, the number of "thumbs up" and the non-stop word overlap with the questions were all identified as potential indicators. Therefore, the enrichment of SM data is clearly a valuable process in a number of scenarios and our techniques will almost certainly be interesting for other application fields as well.

The provision of enriched data is helpful due to the fact that situation assessment has been shown to be very subjective [15]. Thus, information needs to depend on personal feelings, experience and the situation itself [16]. However, wherever information is gathered and analyzed, and information systems are implemented to support this task, there is a common challenge: how to implement information systems to allow both the automatic selection of relevant data and the possibilities for end-users to adapt this automation and enable tailorable quality assessment according to their needs [11]? Enriched data will help us to face this challenge.

\footnotetext{
${ }^{1}$ http://www.w3.org/blog/2014/12/opensocial-foundation-moves-standardswork-to-w3c-social-web-activity/

2 JavaScript Object Notation
} 


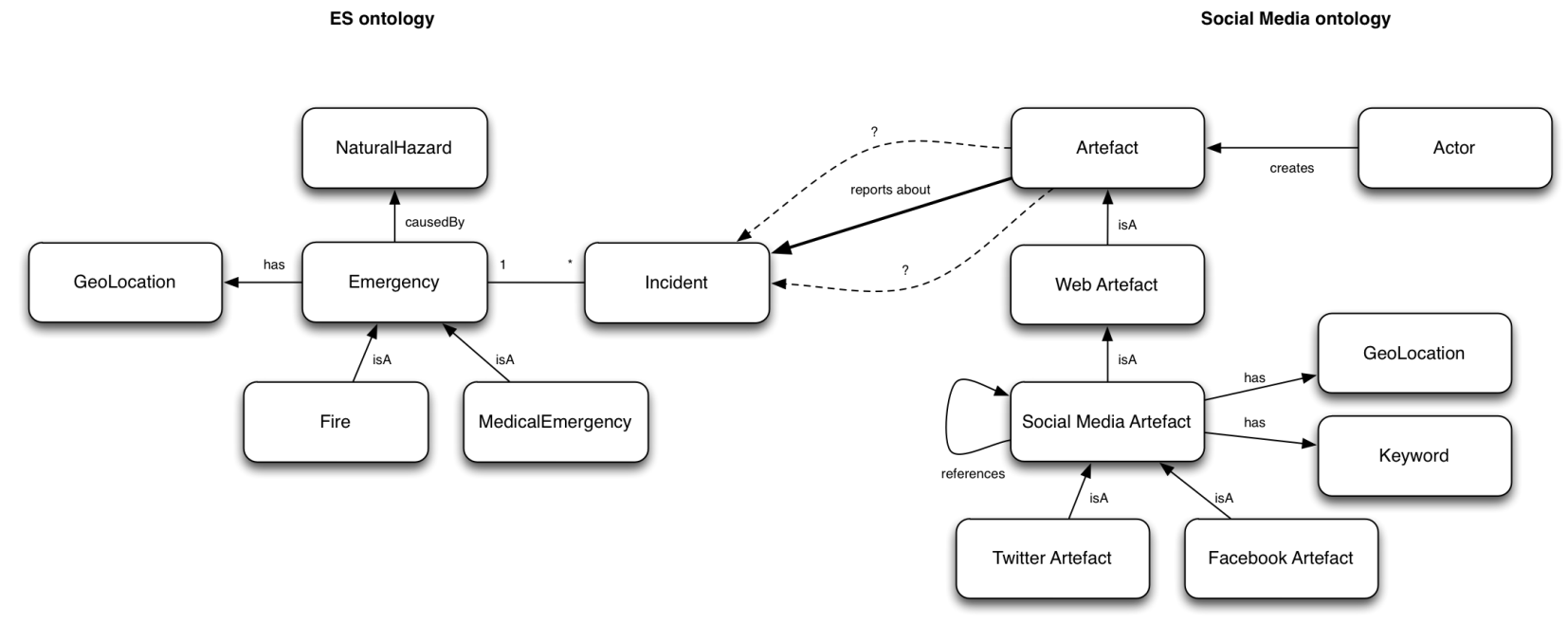

Fig. 2. Approach to build the EmerGent ontology (Example)

\section{Information Mining}

The two stages we have discussed so far provide us with enriched SM data in a common format. This enables us to easily mine the data to obtain the key information for emergency services. During information mining (IM), messages enriched with meta-data become transformed into information objects representing domain-related knowledge.

Many standard IM techniques have been developed and successfully implemented across a range of applications [17], [18]. However, mining SM comes with a set of unique challenges which have been the focus of much research in recent years [19]-[21]. Standard natural language processing (NLP) tools often fail when faced with the non-standard, 'messy' language frequently found in SM [22], [23]. This is compounded by the issues of scalability that are present with SM data. During a crisis there is often a huge surge of related activity online [24], and the mining tools must be able to competently deal with these increased volumes of data.

Many SM messages will contain very similar content, thus we need to remove any duplicate information and consolidate the unique information into related events. This is done using clustering algorithms with various similarity measures [25], [26]. For our system, we are only interested in messages relevant to emergency authorities. In our investigations we have found that searching social media for specific keywords thought to be relevant to emergency services leads to a large amount of irrelevant data from social media. Thus it is essential that we are able to classify messages accordingly. We do so using a Naïve Bayes Classifier, variants of which are commonly used in spam filtering of email. Using these filters vastly reduces the amount of information emergency services need to assess, and ensures that a significantly higher proportion is relevant. As well as removing irrelevant information, we need to extract additional information about an event which will be needed by the user, such as its location and coverage. To do so, we analyze the enriched data as well as using additional NLP techniques on any textual content.
Our combination of mining techniques consolidate multiple SM messages into an information-rich event. However, we need a suitable method to model this event information. It is to this stage in our processing pipeline that we now turn.

\section{Semantic information modelling with ontologies}

During information mining, data is transferred into information that needs to be understood in a domain-specific context. [27] summarizes it as follows: "Semantic technologies have the potential to help people cope better with social mediainduced information overload. Automatic semantic-based methods that adapt to individual's information seeking goals and summarize briefly the relevant SM, could ultimately support information interpretation and decision making over large-scale, dynamic media streams."

The importance of having a common understanding within emergencies, especially in emergency management has been recognized; consequently, different vocabularies, taxonomies and ontologies have been created by multiple agencies involved in emergency prevention, response and recovery processes. On-going research [28], [29] has shown that the need for a common understanding of concepts within and across domains is important to avoid misunderstandings. A further issues is that it is common practice to build an ontology from scratch, which does not tap the existing potential of relevant, domain-related knowledge bases. Thus ontologies are often implicitly tailored to a specific need [30]. To facilitate information exchange with external systems, projects or domains it is necessary that new developments build upon existing standards. Therefore existing information models like $\mathrm{FOAF}^{3}, \mathrm{SIOC}^{4}$ or MOAC ${ }^{5}$ must be considered in order to build an ontology that associates information from SM with domain knowledge.

Our general approach is to reuse and extend existing information models in order to combine extracted emergency

\footnotetext{
${ }^{3}$ Friend of a Fried (http://www.foaf-project.org)

${ }^{4}$ Semantically-Interlinked Online Communities (http://sioc-project.org)

${ }^{5}$ Management of a Crisis (http://www.observedchange.com/moac/ns/)
} 
related content with social media data. The general idea of our approach is shown in Fig. 2. The emergency services domain is shown on the left, to describe a simple emergency situation. Each emergency may have several incidents or reports on incidents. The right side represents the structure of SM data (including some meta-data). Typed relations, so called semantic relations, are used to do the mapping between these two "worlds". In this example there is a relation "reports about" to associate artefacts (a tweet can be a twitter artefact) with an incident. Hence, from a modelling perspective the main steps are:

- Development of a model to describe data from SM in order to understand the structure with all features, properties and constraints in SM. Particularly available meta-data like replies, mentions, references, keywords, timestamps, geo-location etc., has to be structured in a common way to apply IM and information quality methods not only on the content of an SM information object itself. It is necessary to understand the semantics of SM data, because otherwise it would be hard to exploit the full potential of SM, especially in emergency situations.

- Development of a model to describe domain-related information of the emergency services. The purpose is to model emergency-related information like incident descriptions, alerts or requests.

- Build a mapping between domain-related information and information from SM. The mapping approach enables emergency services to comprehend the associations between emergency-related information and processed data from SM (E.g. tweets, Facebook posts).

The ontology will be used to apply semantic analysis on gathered data from SM. This includes the application of further data mining methods to detect patterns, incidents or unusual events as well as the detection of correlations. Another advantage of this approach is that emergency services are not necessarily required to deal with tweets or posts and may work with domain-related information.

\section{E. Information Quality}

With the empowerment of the general public and the abundance of information on SM, fostering information quality (IQ) is central for decision makers to achieve an effective and efficient outcome in the emergency response [31]. Because of the huge amount of data and unclear content quality, the challenge is to provide an automated process and IT-based solution for the assessment.

Very little has been done to examine the quality of data obtained through SM tools and technologies [32]. Although there are concerns about the quality of SM information, understanding of relevant quality attributes and effective means for assessing them is limited. This has raised, for many researchers, the question of the quality of user-generated content in SM [33], especially in the domain of emergency management. Therefore, the challenge is in a first step to define an adequate IQ framework of criteria and relevant indicators influencing the defined criteria (see Fig. 3). Adequateness means relevant criteria to assess the IQ of SM data for emergency service, like timeliness, understandability and believability [34] and also the criterion "trustworthiness" which becomes more and more significant in the context of quality of SM data (e.g. [35], [36]). Initially the defined criteria determine equally the overall value for the IQ.

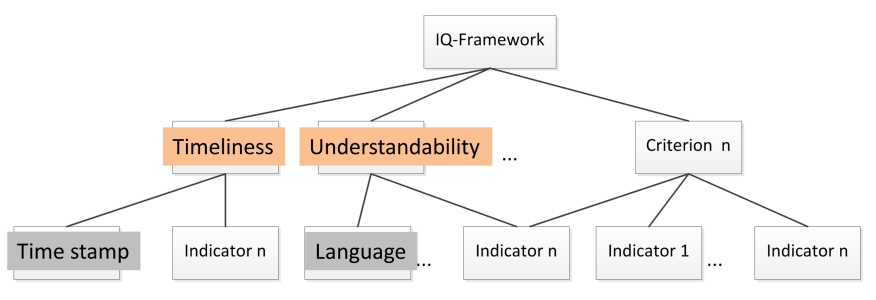

Fig. 3. Structure of IQ framework

But another issue is that something perceived as excellent quality by one user might be considered to be of poor quality by another user [37]. This leads to the challenge to enable a flexible and dynamic assessment of the information on SM. The weighting of the criteria of the IQ framework are initially equally influencing the overall value of the IQ, but can be adjusted based on the preferences of the user. Thereby the concept is to even allow the complete suppression of a criterion.

In addition to the appropriate definitions of criteria and indicators, a further challenge is the determination of the acceptable reference values for the assessment of the indicators. For example, it is necessary to specify the acceptable time frame of the time stamp. Currently, statistics in the literature (e.g., 75\% of the engagement on a Facebook post happens in the first 5 hours) ${ }^{6}$ and requirements and feedback gained during interviews and workshop with end-users from emergency services are taken into account.

Another example is the understandability of a SM message. Appropriate indicators including spelling and post length [38]. The assessment of these indicators (and hence reference values) may change according to the considered SM channel. E.g. Twitter is limited by 140 characters per message and therefore forces users to use short sentences and abbreviations. By assessing these types of indicators it is therefore necessary to consider the source. Individual preferences (e.g., the language) also have an effect on the assessed value of the understandability. Again, the approach is a flexible and useroriented approach, which allows the adjustment of the reference values (e.g., add further languages).

\footnotetext{
${ }^{6} \mathrm{http}: / /$ blog.digitalinsights.in/social-media-users-2014-statsnumbers/05205287.html
} 


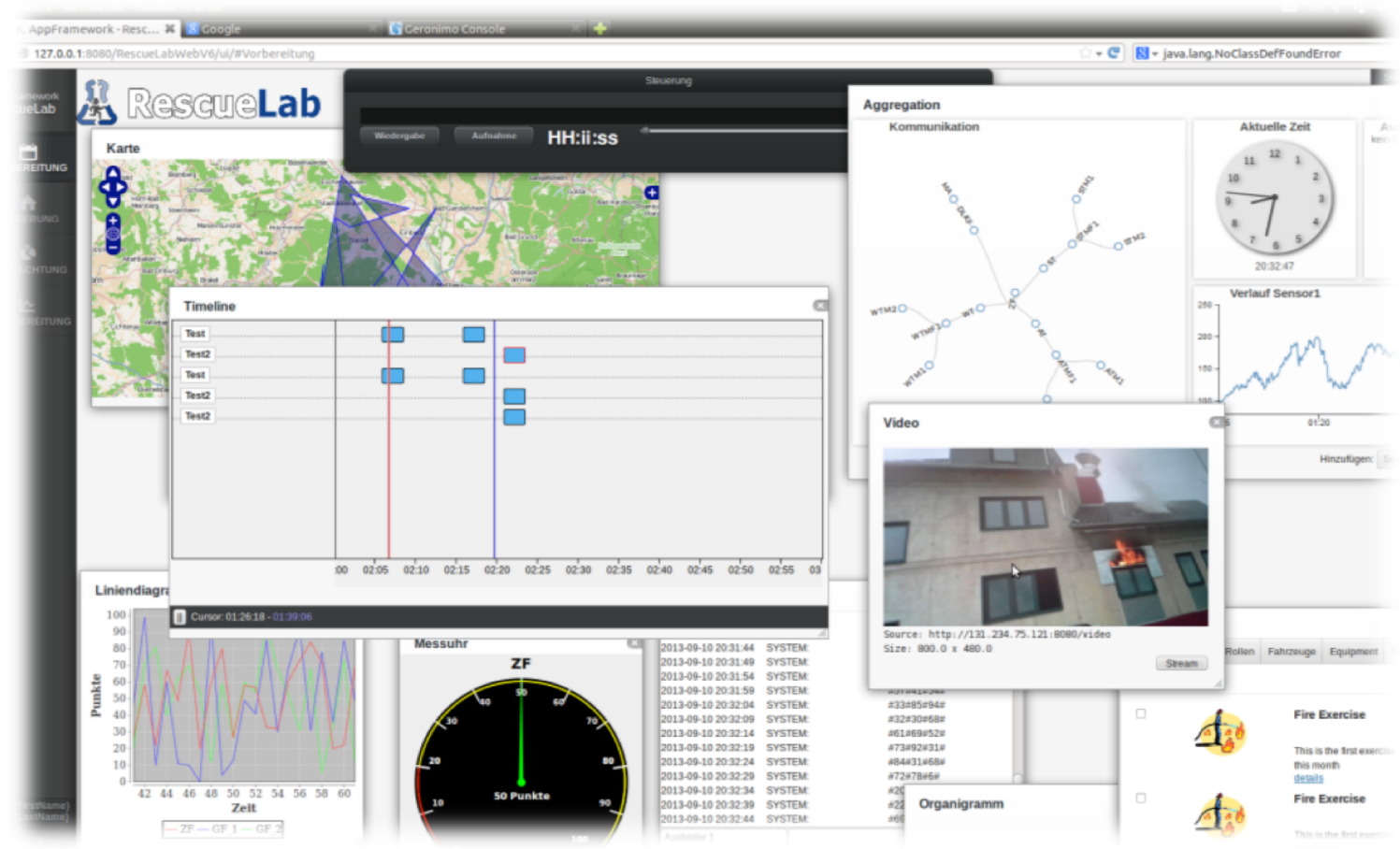

Fig. 4. Exemplary implementation of visualisation techniques [39]

\section{F. Alert Detection}

The alert detection operates on mined and quality-rated information to detect emergency-related events such as "two injured persons detected". An alert has at least a type (e.g. "Injured Person") to describe the context of the event, a timestamp and information on the event source (e.g. the senderID).

The alert detection is always domain-related because involved parties, such as fire or police departments, have different duties. Basically, it acts like a semantic reasoner on an information space to infer logical consequences from asserted facts or axioms. Whenever something in the information space changes, defined rules may become true and new alerts will be generated. There are several existing tools and frameworks, such as Drools ${ }^{7}$, Flora- $2^{8}$ or Jena ${ }^{9}$, available to build a semantic reasoner. However, apart from technical details, the main challenge for the development of suitable alert detection relies on the definition of facts and axioms to define relevant alerts, because of the need of broad domain knowledge.

\section{G. Information Visualisation}

There are several kinds of visualisation techniques for complex datasets (including SM datasets): simple lists, spatial and temporal representations or charts and graph-based visualisations. The project aims at combining such techniques in a layered and modular concept. The high-level visualisation applies very restrictive filters to keep the amount of data as small as possible. The low-level visualisation provides a more detailed view on the data. Three types of user groups require

\footnotetext{
${ }^{7}$ http://www.drools.org/

${ }^{8}$ http://flora.sourceforge.net/

${ }^{9} \mathrm{https} / / /$ jena.apache.org/
}

the entire chain from high-level to low-level visualisations: citizens, emergency services and researchers.

Citizens are expected to be interested in more aggregated information (e.g. as lists and spatial representations). Emergency services will also be interested in the aggregated information, but will want to be able to request more detailed information. For example, if emergency personnel receive an alert, they need the option to investigate the origin or the reason for it. Thus it must be possible to visualise the dependencies between alerts and single posts which initiated an event. These can be temporal representations or graph-based visualisations. Finally, researchers are primarily interested in details and potential relations between data objects. Researchers also include system developers, who want to know about activities inside the system, in order to improve functionalities. This will mainly involve graph-based visualisations and charts.

The visualisation techniques will be implemented in a flexible framework for researchers, as interface for emergency services, and as an app for citizens. Fig. 4 shows the exemplary implementation of different visualization techniques in the framework, which initial version was developed in former projects [39].

\section{DISCUSSION AND CONCLUSION}

In this paper we have outlined a strategy for processing and analysing SM data streams. This set of tools can be used before, during, and after emergencies. We discussed the key steps for an appropriate processing and analysis system: (A) information gathering (incl. data preparation), (B) data enrichment, (C) semantic information modelling with ontologies, (D) information mining, (E) information quality, (F) alert detection and $(\mathrm{G})$ information visualisation. 
There are data exchange formats available, which can be enhanced to fulfil the requirements of the proposed integrated concept. A variety of attributes can be extracted from the heterogeneous SM services and transferred into a homogeneous data format. More complex computations like duplicate and relationship detection between information objects will be done by information mining methods. Information quality assessment will analyse information objects based on a framework of suitable criteria for SM data in emergencies. Alert detection will complete the concept of transferring high volume, but unclear information content into low volume and rich content suitable for emergency services. The information visualisation builds upon a layered and modular concept for three stakeholders with different requirements.

In the next stages of our work, the described concept will be designed and implemented further (as part of an IT-system within the EmerGent project), following the principles of service-oriented architectures. Each sub-process will be designed and implemented as a single (sub-)system component. A central service orchestration component will allow us to edit workflows in a very flexible manner. The system will be tested by emergency services, as well as with mobile apps by emergency services and citizens. To support the analysis of the positive and the negative impact of SM in emergencies, visualisation techniques for SM data will be applied across all user interface prototypes. The system will then be made available to emergency services and citizens to perform deeper evaluation of the project.

\section{ACKNLOWLEDGEMENTS}

The research project EmerGent (www.fp7-emergent.eu) was funded by a grant of the European Union (FP7 No. 608352).

\section{REFERENCES}

[1] C. Reuter, T. Ludwig, M.-A. Kaufhold, and V. Pipek, "XHELP: Design of a Cross-Platform Social-Media Application to Support Volunteer Moderators in Disasters," in Proceedings of the Conference on Human Factors in Computing Systems (CHI), 2015.

[2] L. A. St. Denis, K. M. Anderson, and L. Palen, "Mastering Social Media: An Analysis of Jefferson County's Communications during the 2013 Colorado Floods," in 11th International ISCRAM Conference, 2014, pp. 737-746.

[3] A. L. Hughes, L. A. S. Denis, L. Palen, and K. M. Anderson, "Online Public Communications by Police \& Fire Services during the 2012 Hurricane Sandy," in Proceedings of the Conference on Human Factors in Computing Systems (CHI), 2014.

[4] A. Blandford and B. L. W. Wong, "Situation awareness in emergency medical dispatch," International Journal of Human Computer Studies, vol. 61. pp. 421-452, 2004.

[5] T. Ludwig, C. Reuter, T. Siebigteroth, and V. Pipek, "CrowdMonitor: Mobile Crowd Sensing for Assessing Physical and Digital Activities of Citizens during Emergencies," in Proceedings of the Conference on Human Factors in Computing Systems (CHI), 2015.

[6] Harvard Humanitarian Initiative, "Disaster Relief 2.0: The future of information sharing in humanitarian emergencies," Harvard Humanitarian Initiative, 2010. .

[7] C. Reuter, A. Marx, and V. Pipek, "Crisis Management 2.0: Towards a systematization of Social Software Use in Crisis situations," Int. J. Inf. Syst. Cris. Response Manag., vol. 4, no. 1, pp. 1-16, 2012.

[8] C. Reuter and S. Scholl, "Technical Limitations for Designing Applications for Social Media," in Mensch \& Computer 2014: Workshopband, 2014, pp. 131-140.
[9] S. Chaudhuri, "What Next? A Half-Dozen Data Management Research Goals for Big Data and the Cloud," Proc. 31st Symp. Princ. Database Syst., pp. 1-4, 2012.

[10] F. Abel, Q. Gao, G. J. Houben, and K. Tao, "Semantic enrichment of twitter posts for user profile construction on the social web," Lect. Notes Comput. Sci. (including Subser. Lect. Notes Artif. Intell. Lect. Notes Bioinformatics), vol. 6643 LNCS, pp. 375-389, 2011.

[11] C. Reuter, T. Ludwig, M. Ritzkatis, and V. Pipek, "Social-QAS: Tailorable Quality Assessment Service for Social Media Content," in Proceedings of the International Symposium on End-User Development (IS-EUD). Lecture Notes in Computer Science, 2015.

[12] C. Reuter, M. Ritzkatis, and T. Ludwig, "Entwicklung eines SOAbasierten und anpassbaren Bewertungsdienstes für Inhalte aus sozialen Medien,” in Informatik 2014 - Big Data - Komplexität meistern, 2014.

[13] C. Diamantini, D. Potena, A. Sabelli, and S. Scattolini, "An integrated system for social information discovery," in Collaboration Technologies and Systems (CTS), 2014 International Conference on, 2014, pp. 353360.

[14] E. Agichtein, C. Castillo, and D. Donato, "Finding High-Quality Content in Social Media," in Proceedings of the ACM Conference on Web Search and Data Mining, 2008, pp. 183-193.

[15] C. Rizza, Â. Pereira, and P. Curvelo, "Do-it-yourself JusticeConsiderations of Social Media use in a Crisis Situation: The Case of the 2011 Vancouver Riots," in Proceedings of the Information Systems for Crisis Response and Management (ISCRAM), 2013, pp. 411-415.

[16] T. Ludwig, C. Reuter, and V. Pipek, "Social Haystack: Dynamic Quality Assessment of Citizen-Generated Content in Social Media during Emergencies," Trans. Hum. Comput. Interact., vol. 21, no. 4, 2015.

[17] M. J. Zaki and W. Meira, Data Mining and Analysis: Fundamental Concepts and Algorithms. Cambridge: Cambridge University Press, 2014.

[18] M. Kantardzic, Data Mining: Concepts, Models, Methods, and Algorithms, 2nd ed. Wiley-Blackwell, 2011.

[19] K. Gimpel, N. Schneider, B. O'Connor, D. Das, D. Mills, J. Eisenstein, M. Heilman, D. Yogatama, J. Flanigan, and N. Smith, "Part-of-speech tagging for Twitter: annotation, features, and experiments," in Proceedings of the 49th Annual Meeting of the Association for Computational Linguistics: Human Language Technologies: short papers, 2011, vol. 2, pp. 42-47.

[20] M. Imran, S. Elbassouni, C. Castillo, F. Diaz, and P. Meier, "Extracting information nuggets from disaster-related messages in social media," in Proceedings of the 10th international ISCRAM confernce, 2013.

[21] A. Ritter, S. Clark, Mausam, and E. O, "Named Entity Recognition in Tweets: An Experimental Study," in EMNLP '11 Proceedings of the Conference on Empirical Methods in Natural Language Processing, 2011, pp. 1524-1534.

[22] J. Eisenstein, "What to do about bad language on the internet," in Proceedings of NAACL-HLT 2013, 2013, pp. 359-369.

[23] X. Liu, M. Zhou, X. Zhou, Z. Fu, and F. Wei, "Joint inference of named entity recognition and normalization for tweets," in Proceedings of the ACL, 2012.

[24] J. D. Fraustino, L. Brooke, and Y. Jin, "Social media use during disasters: a review of the knowledge base and gaps," 2012.

[25] J. Yin, A. Lampert, M. Cameron, B. Robinson, and R. Power, "Using social media to enhance emergency situation awareness," Intell. Syst. IEEE, vol. 27, no. 6, pp. 52-59, 2012.

[26] H. Becker, M. Naaman, and L. Gravano, "Event identification in social media," in Twelfth International Workshop on the Web and Databases, 2009.

[27] K. Bontcheva and D. Rout, "Making sense of social media streams through semantics: A survey," Semant. Web, vol. 5, pp. 373-403, 2014.

[28] A. Galton and M. Worboys, "An ontology of information for emergency management,” Int. Conf. Cris. Response Manag., vol. 8, no. May, pp. 1$10,2011$.

[29] K. Grolinger, K. Brown, and M. Capretz, "From Glossaries to Ontologies: Disaster Management Domain,” 2011.

[30] E. P. Bontas, M. Mochol, and R. Tolksdorf, "Case Studies on Ontology Reuse," in Proceedings of I-KNOW'05, 2005, pp. 345-353. 
[31] G. E. Jensen, "Key criteria for information quality in the use of online social media for emergency management in New Zealand," Victori a University of Wellington, 2012.

[32] G. Shankaranarayanan, B. Iyer, and D. Stoddard, "Quality of Social Media Data and Implications of Social Media for Data Quality," in International Conference on Information Quality (ICIQ), 2012, pp. 311325.

[33] F. F. Emamjome, A. A. Rabaa'i, G. G. Gable, and W. Bandara, "Information quality in social media : a conceptual model," in PACIS 2013 The Pacific Asia Conference on Information Systems, 2013.

[34] T. Friberg, S. Prödel, and R. Koch, "Analysis of information quality criteria in crisis situation as a characteristic of complex situations," in Proceedings of the 15th International Conference on Information Quality, 2010.

[35] S. ten Kate, "Trustworthiness within Social Networking Sites: A study on the intersection of HCI and Sociology," University of Amsterdam, 2009.
[36] J. R. C. Nurse, S. S. Rahman, S. Creese, M. Goldsmith, and K. Lamberts, "Information quality and trustworthiness: A topical state-ofthe-art review," in International Conference on Computer Applications and Network Security (ICCANS), 2011.

[37] K. Chai, V. Potdar, and T. Dillon, "Content quality assessment related frameworks for social media," in Lecture Notes in Computer Science (including subseries Lecture Notes in Artificial Intelligence and Lecture Notes in Bioinformatics), 2009, vol. 5593 LNCS, pp. 791-805.

[38] W. Weerkamp and M. de Rijke, "Credibility improves topical blog post retrieval," ACL-08 HLT 46th Annu. Meet. Assoc. Comput. Linguist. Hum. Lang. Technol. Proc. Conf., Jun. 2008.

[39] J. Pottebaum, R. Marterer, T. Friberg, and R. Koch, “Anforderungen an die IT-Unterstützung in Einsatzübungen der nicht-polizeilichen Gefahrenabwehr," in Informatik 2013 - Informatik angepasst an Mensch, Organisation und Umwelt, 2013. 